\title{
Apoptosis induced by sonodynamic therapy in human osteosarcoma cells in vitro
}

\author{
XING LIU ${ }^{1}$, WEI LI ${ }^{1}$, SHUO GENG ${ }^{1}$, QING-GANG MENG ${ }^{2}$ and ZHENG-GANG BI ${ }^{1}$ \\ ${ }^{1}$ Department of Orthopaedic Surgery, The First Affiliated Hospital of Harbin Medical University; \\ ${ }^{2}$ Department of Orthopedic Surgery, The First Hospital of Harbin City, Harbin Medical University, \\ Harbin, Heilongjiang 150001, P.R. China
}

Received May 27, 2014; Accepted February 17, 2015

DOI: $10.3892 / \mathrm{mmr} .2015 .3479$

\begin{abstract}
The aim of the present study was to investigate the potential effect of hematoporphyrin monomethyl ether-sonodynamic therapy (HMME-SDT) on MG-63 osteosarcoma cells. The HMME concentration was kept constant at $20 \mu \mathrm{g} / \mathrm{ml}$ and the MG-63 osteosarcoma cell line was exposed to ultrasound with an intensity of $1.0 \mathrm{~W} / \mathrm{cm}^{2}$ for $30 \mathrm{sec}$. Cell cytotoxicity was quantified using an MTT assay $6 \mathrm{~h}$ after HMME-SDT. The intracellular localization of HMME was imaged using inverted confocal laser scanning microscopy. Apoptosis was investigated using flow cytometry with Annexin V-fluorescein isothiocyanate and propidium iodine staining. The cytotoxicity of HMME-mediated sonodynamic action on MG-63 cells was significantly higher than that of other treatments, including ultrasound alone, HMME alone and sham treatment. Flow cytometry demonstrated that HMME-SDT action markedly enhanced the apoptotic rate of MG-63 cells. The mechanisms of apoptosis were analyzed by measuring the protein expression of poly ADP-ribose polymerase (PARP), cleaved PARP, procaspase-3, cleaved caspase- 3 and cleaved caspase- 9 . The data demonstrated that HMME-SDT action markedly induced the apoptosis of MG-63 cells.
\end{abstract}

\section{Introduction}

Osteosarcoma (OS) is the most common type of primary malignant bone tumor, and predominantly occurs in infants (1). With the development of novel chemotherapy protocols, surgical excision and radiological staging, 5-year survival rates and cure rates have increased to $60-80 \%$ in patients with localized disease (2). However, problems with

Correspondence to: Dr Zheng-Gang Bi, Department of Orthopedic Surgery, The First Affiliated Hospital of Harbin Medical University, 23 You Zheng, Harbin, Heilongjiang 150001, P.R. China

E-mail: 15846537651@163.com

Key words: sonodynamic therapy, apoptosis, osteosarcoma, hematoporphyrin monomethyl ether chemotherapy remain, particularly the frequent development of drug resistance (3). Therefore, there remains a requirement for the development of alternative methods for the treatment of OS. In recent years, the use of SDT has been widely accepted as a promising therapeutic strategy to prevent the development and recurrence of malignant diseases.

Sonodynamic therapy (SDT) is a non-invasive approach for the treatment of diseases based on the synergistic effects of low intensity ultrasound and sonosensitization (4-6). The ultrasound energy can be focused on targeted tissues to induce local cytotoxicity by activating sonosensitizers with minimal damage to healthy tissues $(7,8)$. Recently, SDT has been widely used in the treatment of tumors and has been demonstrated to mediate apoptosis in numerous experimental systems in vitro or in vivo. SDT has been shown to induce cell death by apoptosis in a number of human tumor cell lines, such as liver, oral, leukemia, lung and colon cancer cell lines (9).

Therefore, SDT has great advantage as a targeted cancer therapy. The ultimate goal of anticancer therapy is to kill cancer cells rapidly and effectively. In the present study, the effects of SDT on the MG-63 human osteosarcoma cell line were investigated using in vitro assays for proliferation and apoptosis. The mechanisms of apoptosis were analyzed by measurement of the protein expression of poly ADP-ribose polymerase (PARP), cleaved PARP, procaspase-3, cleaved caspase-3 and cleaved caspase-9.

\section{Materials and methods}

Cell culture. The MG-63 human osteosarcoma cell line was obtained from the American Type Culture Collection (Rockville, MD, USA). The cell line was cultured in Dulbecco's modified Eagle's medium (DMEM, Life Technologies, Inc., Grand Island, NY, USA) supplemented with $10 \%$ fetal bovine serum (FBS, Hyclone, NY, USA), $100 \mathrm{U} / \mathrm{ml}$ penicillin and $100 \mu \mathrm{g} / \mathrm{ml}$ streptomycin (Wuhan Boster Biological Technology, Ltd., Wuhan, China) at $37^{\circ} \mathrm{C}$ in a humidified atmosphere of $5 \%$ $\mathrm{CO}_{2}$ in air. Experiments were conducted in logarithmic growth phase cells.

Ultrasonic exposure system and SDT treatment protocols. The ultrasonic exposure system (Fig. 1) used in our study was multifunctional physiotherapy ultrasonic equipment (Tianshi 
Technologies Ltd Co., Beijing, China). Using this device, ultrasound at $1 \mathrm{MHz}$ could be produced. The transducer (diameter, $2.5 \mathrm{~cm}$; centre frequency, $1.0 \mathrm{MHz}$; duty factor, $10 \%$; and repetition frequency, $100 \mathrm{~Hz}$ ) was placed upward at the bottom of a water tank. The tank was filled with degassed water and the inner surface was padded with ultrasound-absorbing materials (Tianshi Technologies Co., Ltd., Beijing, China) to minimize the wave reflection. The spatial average and temporal average intensities (ISATA) was measured with a stainless-steel ball radiometer (diameter, $0.32 \mathrm{~cm}$; Tianshi Technologies Co., Ltd.) over $30 \mathrm{sec}$. The output intensity was $1 \mathrm{~W} / \mathrm{cm}^{2}$ during exposure. MG-63 cells were seeded in $35-\mathrm{mm}$ petri dishes and placed in the degassed water at a distance of $\sim 5.2 \mathrm{~cm}$ from the ultrasonic transducer. During exposure, the temperature of the water was maintained at $37^{\circ} \mathrm{C}$. During the sonication procedure, the temperature of the solution inside the petri dishes was measured prior to and following ultrasound treatment with a digital thermometer, and no significant variation of temperature was detected $\left( \pm 0.5^{\circ} \mathrm{C}\right)$.

HMME was obtained from Yingfa Kangmei Ltd. Co., (Beijing, China). The cells were divided into the following four groups: Sham group, which received no treatment; the HMME group, which was treated with $20 \mu \mathrm{g} / \mathrm{ml}$ HMME alone; the ultrasound group treated with $1 \mathrm{~W} / \mathrm{cm}^{2}$ ultrasound alone and the ultrasound + HMME (SDT) group, which was treated with $1 \mathrm{~W} / \mathrm{cm}^{2}$ ultrasound plus $20 \mu \mathrm{g} / \mathrm{ml}$ HMME.

Cell viability assays. Cells were seeded into $35-\mathrm{mm}$ petri dishes (Bogoo Biotechnology Company, Shanghai, China) and incubated with different concentrations of $\operatorname{HMME}(0,5,10,20,30$, 40 and $50 \mu \mathrm{g} / \mathrm{ml}$ ) for $3 \mathrm{~h}$ in the dark. They were then exposed to ultrasound for $30 \mathrm{sec}$. After SDT (6 h), the cell survival rate was determined by an MTT assay. The MTT assay provides a fast and simple method to evaluate the viability of the cells in the SDT (10). This assay was performed as a regular procedure and the absorbance at $570 \mathrm{~nm}$ was recorded using a microplate reader (BIO-TEK ELx800, BioTek, San Diego, CA, USA) against the reference value at $690 \mathrm{~nm}$. Results were expressed as a percentage of the control.

Intracellular localization of hematoporphyrin monomethyl ether. MG-63 cells were incubated with $20 \mu \mathrm{g} / \mathrm{ml}$ HMME for $4 \mathrm{~h}$, then co-loaded with $10 \mathrm{nM}$ Mito Tracker Green (MT-G, Molecular Probes, Invitrogen Life Technologies, Carlsbad, CA, USA) and $1 \mu \mathrm{g} / \mathrm{ml}$ Hoechst 33342 (Ho; Molecular Probes, Invitrogen Life Technologies). After loading, cells were washed with phosphate-buffered saline (PBS) and imaged using an inverted confocal laser scanning microscope (TCS SP5Leica, Wetzlar, Germany). In the multi-channel imaging, photomultiplier sensitivities and offsets were set to a level at which bleed through effects from one channel to another were negligible.

Cell apoptosis assays. Cell apoptosis was detected using the Annexin V-fluorescein isothiocyanate (FITC)/propidium iodide (PI) apoptosis detection kit (KeyGen, Nanjing, China). In brief, $1 \times 10^{5}$ treated cells were harvested following trypsinization and centrifugation at $111.8 \mathrm{xg}$ for $5 \mathrm{~min}$. Cells were washed with PBS and resuspended in $500 \mathrm{ml}$ binding buffer, to which $5 \mathrm{ml}$ Annexin V-FITC was added. After a $5 \mathrm{~min}$

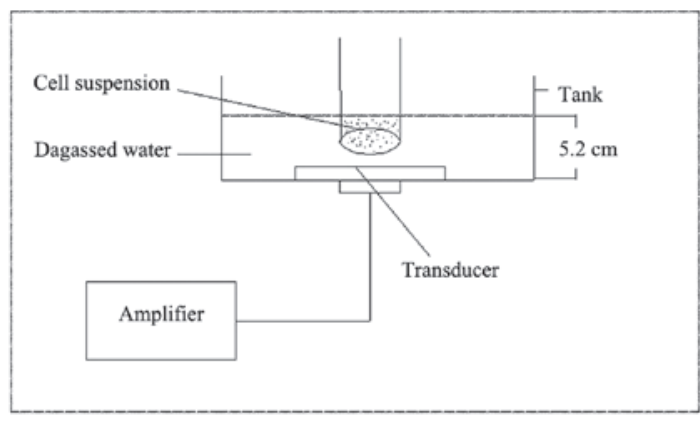

Figure 1. Ultrasonic exposure system.

incubation, $5 \mathrm{ml}$ PI was added, followed by a 5 min incubation in the dark. Apoptotic cells were analyzed by flow cytometry (FC500 system; Beckman Coulter, Fullerton, CA, USA).

Western blot analysis. PARP, cleaved PARP, procaspase-3, cleaved caspase- 3 and cleaved caspase- 9 were measured by western blot analysis. SDS-PAGE and immunoblotting were conducted according to set standard procedures.

Briefly, cells were harvested and collected by centrifugation at $111.8 \mathrm{x}$ g for $5 \mathrm{~min}$, then precooling lysate was added (containing $0.25 \%$ sodium deoxycholate, $1 \%$ Triton X-100, $1 \%$ Nonidet P-40, $5 \mathrm{mM}$ EDTA, $10 \mu \mathrm{g} / \mathrm{ml}$ leupeptin, $10 \mu \mathrm{g} / \mathrm{ml}$ aprotinin and $1 \mathrm{mM}$ phenylmethylsulfonyl fluoride; all from Sigma-Aldrich, St. Louis, MO, USA) on ice. The cells were centrifuged at $111.8 \mathrm{x} \mathrm{g}$ for $5 \mathrm{~min}$, and the supernatant was collected, followed by the addition of $5 \mathrm{X}$ loading buffer (containing 10\% SDS, 5\% $\beta$-mercaptoethanol, $15 \%$ glycerol, $0.01 \%$ bromophenol blue and $200 \mathrm{mM}$ Tris-HCl; $\mathrm{pH}$ 6.7) in boiling water for $5 \mathrm{~min}$ and separated on a $10-15 \%$ linear SDS polyacrylamide gel. Then, the proteins were transferred onto nitrocellulose filter membranes (Millipore, Billerica, MA, USA). Membranes were incubated at room temperature for $2 \mathrm{~h}$ and probed at $4^{\circ} \mathrm{C}$ overnight with mouse monoclonal PARP (cat. no. sc-56197), mouse monoclonal cleaved PARP (cat. no. sc-56196), mouse monoclonal procaspase-3 (cat. no. sc-7272), rabbit monoclonal cleaved caspase-3 (cat. no. sc-22171-R), mouse monoclonal cleaved caspase-9 (cat. no. sc-70505) as primary antibodies all purchased from Santa Cruz Biotechnology, Inc. (San Diego, CA, USA). Membranes were probed with a goat anti-rabbit antibody conjugated with secondary antibody (Beijing Zhongshan Golden Bridge Biotechnology Co., Ltd., Beijing, China) for $1 \mathrm{~h}$ and visualized by enhanced chemiluminescence (Tianshi Technologies Co., Ltd.).

Statistical analysis. Statistical analysis was conducted using SPSS 16.0 statistical software (SPSS, Inc., Chicago, IL, USA). All values are expressed as the mean \pm standard error of the mean. Differences between the treated cells and control were assessed with one-way analysis of variance. $\mathrm{P}<0.05$ was considered to indicate a statistically significant difference.

\section{Results}

HMME-SDT inhibits the proliferation of MG-63 cells. Cell viability was detected by an MTT assay. The survival rate 
A

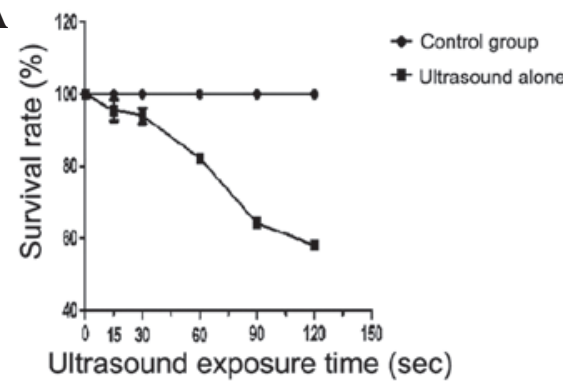

B

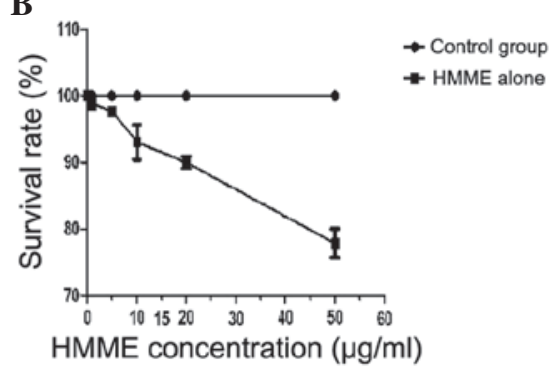

C

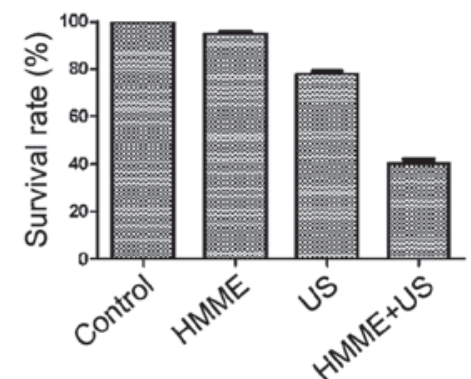

Figure 2. HMME-sonodynamic therapy in MG-63 cells. (A) Ultrasound exposure time, (B) HMME concentration and (C) viability of MG-63 cells after different treatments. Control, no treatment; HMME, $20 \mu \mathrm{g} / \mathrm{ml}$ HMME treatment alone; ultrasound, $1 \mathrm{~W} / \mathrm{cm}^{2}$ ultrasound treatment alone; Ultrasound+HMME, $1 \mathrm{~W} / \mathrm{cm}^{2}$ ultrasound plus $20 \mu \mathrm{g} / \mathrm{ml}$ HMME. P<0.01 versus the sham, HMME and Ultrasound groups. HMME, hematoporphyrin monomethyl ether; US, ultrasound.

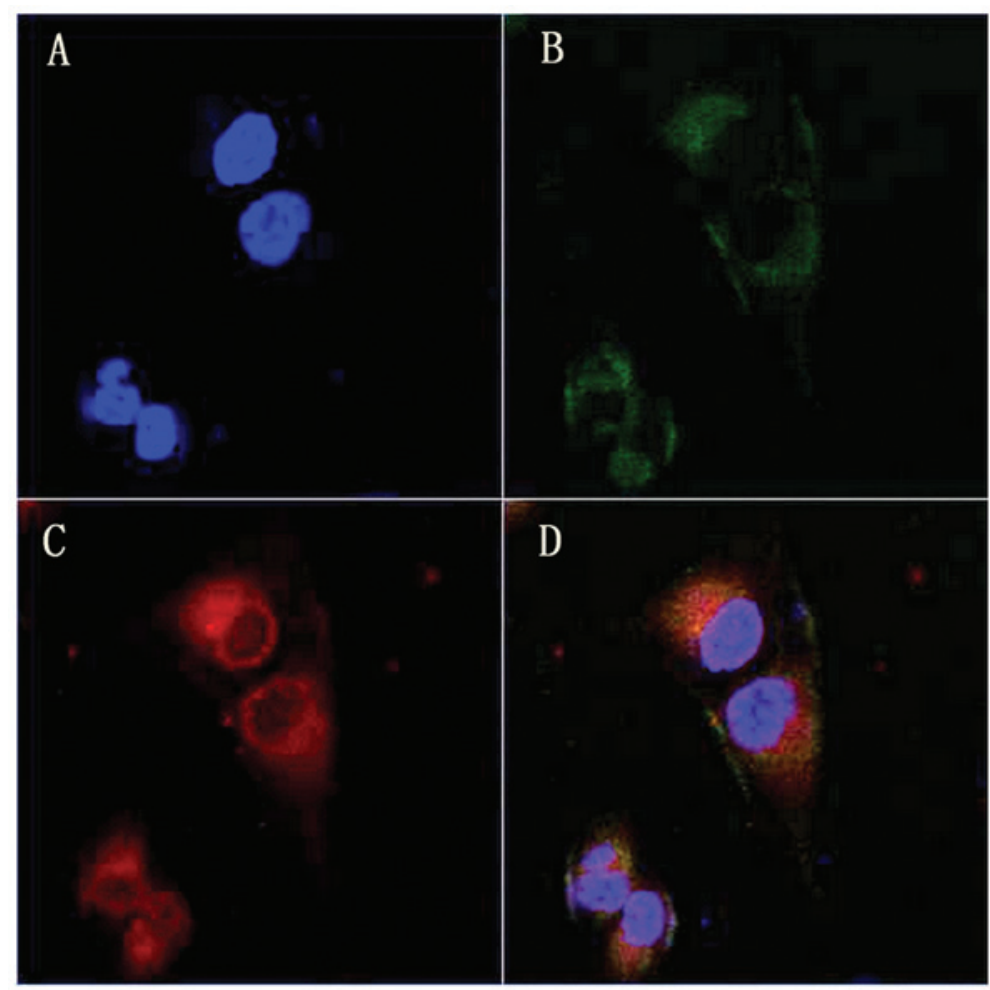

Figure 3. Intracellular localization of HMME. (A) Hoechst 33342 blue fluorescence (magnification x400); (B) MT-G green fluorescence (magnification x400); (C) HMME fluorescence (magnification X400); (D) Hoechst 33342 blue fluorescence, MT-G green fluorescence and HMME fluorescence (magnification x400). HMME, hematoporphyrin monomethyl ether; MT-G, mito tracker green.

of MG-63 cells decreased with HMME concentration and ultrasonic irradiation time increased. As shown in Fig. 2A, the survival rate was significantly decreased with the increase in ultrasound exposure time. As shown in Fig. 2B, the survival rate was significantly decreased with increasing concentration of HMME.

As shown in Fig. 2C, cell survival in $20 \mu \mathrm{g} / \mathrm{ml} \mathrm{HMME}$ alone was $94.70 \pm 0.58 \%$ compared with control, showing no obvious cytotoxic effect on MG-63 cells. When cells were treated with $1.0 \mathrm{~W} / \mathrm{cm}^{2}$ ultrasound, the survival rate of cells was $77.68 \pm 0.89 \%$. However, when cells were treated with ultrasound in the presence of $20 \mu \mathrm{g} / \mathrm{ml} \mathrm{HMME}$, cell survival decreased to $40.18 \pm 1.35 \%$ ( $\mathrm{P}<0.01)$, demonstrating a synergistic enhancement of sonochemically induced cytotoxicity.
Intracellular localization of HMME. After $4 \mathrm{~h}$ incubation with HMME, cells were co-loaded with the mitochondria probe MT-G (green) and nuclear probe Ho (blue). As shown in Fig. 3, the HMME fluorescence (red) corresponded well with MT-G green fluorescence but without any Ho blue fluorescence overlap, indicating that HMME predominantly accumulated in the mitochondria of MG-63 cells.

HMME-SDT induces apoptosis in MG-63 cells. Flow cytometry with Annexin V (Annexin V-FITC) and PI staining has been regarded as an important way to distinguish early apoptosis from late apoptosis or necrosis (11). In the present study, flow cytometry showed that HMME-SDT significantly increased the apoptosis of the MG-63 cell population. Fig. 4 

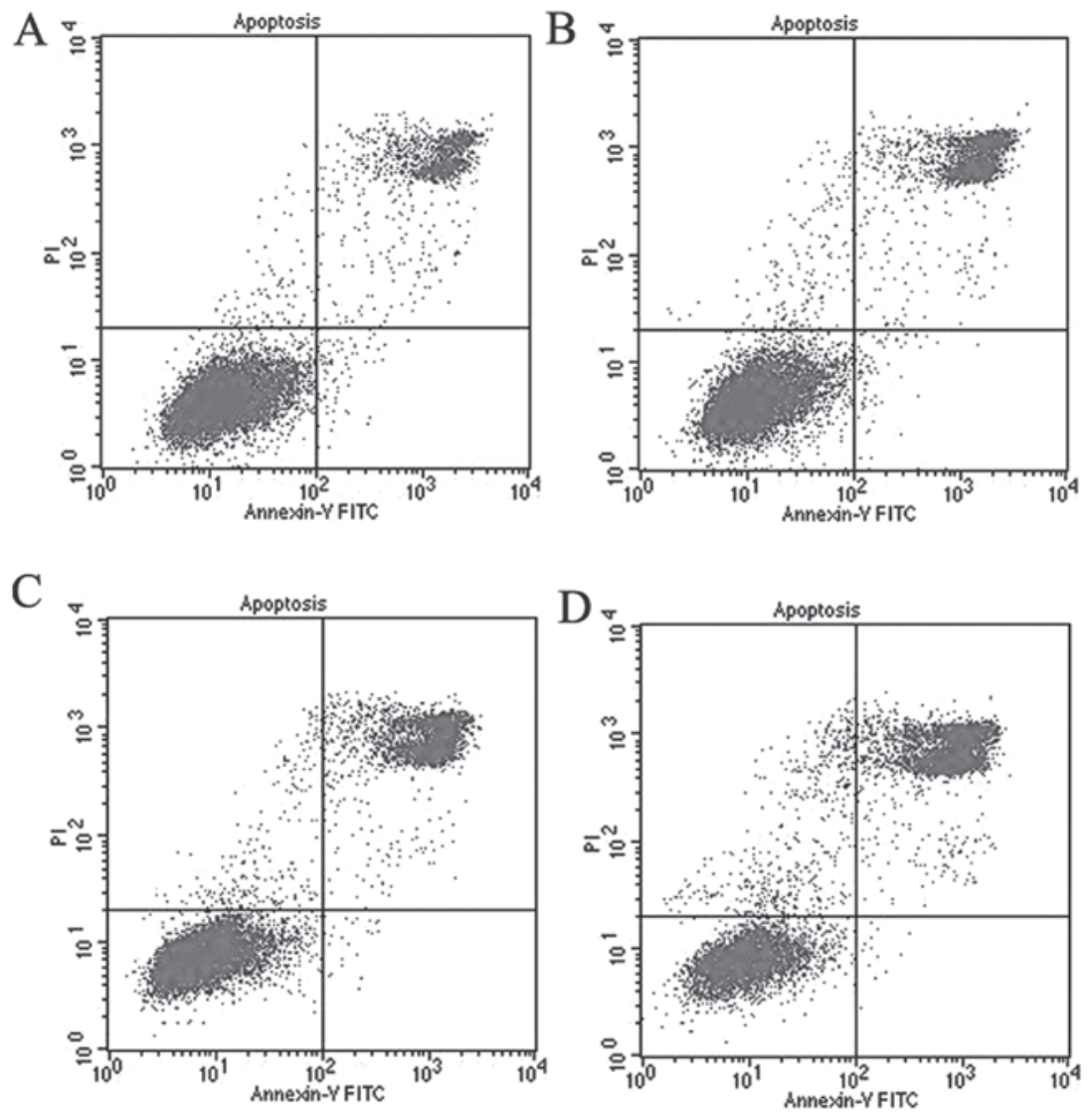

Figure 4. Apoptosis of MG-63 cells was analyzed $12 \mathrm{~h}$ after sonodynamic therapy with HMME $(20 \mu \mathrm{g} / \mathrm{ml})$ under ultrasound sonication $(1.7 \mathrm{MHz})$ with the intensity of $1.0 \mathrm{~W} / \mathrm{cm}^{2}$ for $30 \mathrm{sec}$ using flow cytometry with Annexin V-FITC and propidium iodide staining. (A) sham radiation, (B) HMME alone, (C) ultrasound alone, (D) HMME-mediated sonodynamic therapy. HMME, hematoporphyrin monomethyl ether; FITC, fluorescein isothiocyanate.

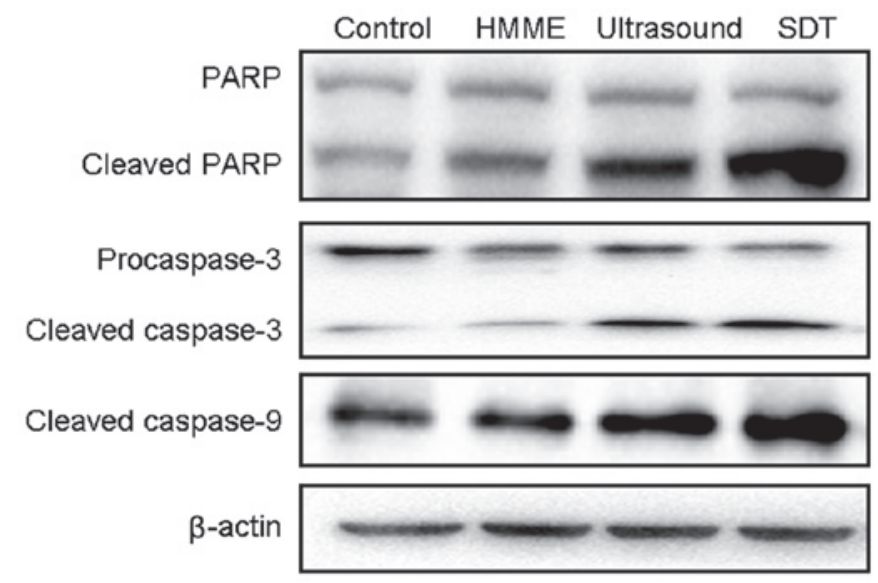

Figure 5. Western blotting analysis of PARP, cleaved PARP, procaspase-3, cleaved caspase-3, cleaved caspase-9. PARP, poly ADP-ribose polymerase.

shows the MG-63 cells $12 \mathrm{~h}$ after sham treatment, $11.49 \%$ of the cells had undergone apoptosis. Following treatment with HMME alone, $19.02 \%$ of the MG-63 cells had undergone apoptosis while following ultrasound treatment alone $23.58 \%$ had undergone apoptosis. However, in the MG-63 cells treated with HMME-SDT, there was a significant increase in the percentage of cells that had undergone apoptosis to $43.56 \%$ at the intensity of $1.0 \mathrm{~W} / \mathrm{cm}^{2}$ for $30 \mathrm{sec}$. These results showed that the sonodynamic action of HMME could effectively induce the apoptosis of OS cells.

Protein expression detected by western blot analysis. Increasing evidence suggests that changes in matrix metalloproteinases are associated with apoptosis (12-14). In a number of experimental systems, SDT has been shown to induce apoptosis. Therefore, the present study aimed to detect several key apoptosis related proteins, such as PARP, cleaved PARP, procaspase-3, cleaved caspase- 3 and cleaved caspase-9, to assess whether MG-63 cells can be induced by SDT.

Cleaved PARP is a classical substrate of activated caspase-3, and it can often be considered as a marker of apoptosis; thus, it was used to indicate the occurrence of apoptosis. PARP cleavage occurs at the early stages of apoptosis, as well as the later stages. Fig. 5 shows that cleaved PARP significantly increased following sonodynamic therapy when compared with that in the ultrasound alone or HMME alone group. Similarly, little cleaved caspase-9 (caspase-9 activation) was measured in either the HMME alone or ultrasound irradiation group, but typical $35 \mathrm{kDa}$ caspase- 9 cleavage was observed, and accompanied by a decrease in the procaspase-9 level. In addition, ultrasound irradiation in the presence of HMME resulted in more obvious caspase- 3 activation compared with the control, accompanied by the decreased level of procaspase-3. These results showed that sonodynamic therapy could induce apoptotic cell death of MG-63 cells, which is caspase dependent. 


\section{Discussion}

Apoptosis is a complex mechanism that regulates cell numbers and is essential throughout the life of all metazoan animals. Caspase activation is the most fundamental pathway among different types of biochemical events in cells undergoing apoptosis (15-20). The induction of apoptosis may be the most effective defense against cancer (20). Recent studies have revealed that low intensity ultrasound combined with sonosensitizers can induce apoptosis in a number of cancer cell types (21-26). The majority of those studies are irradiated by selecting cell types, sonosensitizers and the ultrasound exposure parameters (21-26). This study demonstrated that SDT therapy mediated by HMME has significant antitumor effects on MG-63 human osteosarcoma cells.

The localization of sonosensitizer is important in the treatment of tumors, due to the short half life and short diffusion distance of the majority of radicals derived from the sonosensitizer produced during SDT (27). In order to detect the intracellular localization of HMME in MG-63 cells, cells were co-loaded with HMME, MT-G mitochondria probe and Ho nucleus dye, and their corresponding fluorescence was imaged by inverted confocal laser scanning microscopy. Fig. 3 shows that the fluorescence of HMME corresponded well with that of MT-G but not with Ho, which suggests that HMME is predominantly localized in the mitochondria of MG-63 cells. The cell survival rate was then detected by MTT $6 \mathrm{~h}$ following SDT treatment. Data as shown in Fig. 2B shows that the proposed ultrasound-induced cell damage was markedly enhanced by $20 \mu \mathrm{g} / \mathrm{ml}$ HMME $(\mathrm{P}<0.01)$, whereas HMME alone did not exhibit significant cytotoxic effects on MG-63 cells. Fig. 5 shows that cleaved PARP significantly increased after sonodynamic therapy when compared with treatment with ultrasound alone or HMME alone. Similarly, little cleaved caspase-9 (caspase-9 activation) was measured in either the HMME alone or ultrasound irradiation group, but typical $35 \mathrm{kDa}$ caspase- 9 cleavage was measured, and was accompanied by a decrease in the procaspase- 9 level. In addition, ultrasound irradiation with the presence of HMME resulted in more notable caspase-3 activation compared with the control, accompanied by a decrease in the level of procaspase-3.

Mitochondrial damage may be the predominant reason for HMME-SDT-induced cytotoxicity as HMME primarily accumulated in the mitochondria of MG-63 cells, suggesting that the mitochondria are the target of SDT. As the major energy generators, mitochondrial-mediated apoptosis occurs in response to a wide range of stimuli.

There are two major apoptotic pathways: The external (receptor-mediated) and the intrinsic (mitochondria-mediated). The intrinsic pathway of apoptosis occurs through internal and external stimulation, including a number of mediators, which can promote or inhibit the process (28). The most representative regulators of the mitochondria-mediated pathway are p53, an inducer of apoptosis, and Bcl-2, a molecule with the opposite effect (29-31). The mitochondria-caspase signaling pathway was activated following SDT and ultrasound to promote the expression of pro-apoptotic proteins, such as caspase- 3 and Bax in cancer cells (12).
In another study, histidine significantly reduced the generation of nitrogen oxides and activation of caspase-3 during sonodynamically-induced apoptosis, suggesting that active species, such as singlet oxygen are important in the sonodynamic induction of apoptosis (32). It has been reported that SDT induced apoptosis in cancer cells partially through a $\mathrm{Ca}^{2+}$ dependent pathway. SDT can induce cell apoptosis through ion channels. The intensity of bubble activity determines intracellular $\mathrm{Ca}^{2+}$ level (33) and is closely associated with cell membrane damage and repair. In addition, certain cells maintain high levels of $\mathrm{Ca}^{2+}$ long after ultrasound exposure, which indicates the complete loss of cell membrane integrity (34). An increase in calcium level is the key process underlying cell apoptosis (35).

SDT affects the expression of numerous genes which provides novel insights into the molecular mechanisms of SDT for the treatment of tumors (36).

In conclusion, this study shows that apoptosis participates in SDT-induced cell death in MG-63 cells. The relative percentages of cells undergoing apoptosis following SDT could be experimentally manipulated. The existing research results can provide important insight into SDT-induced cell apoptosis and also indicated that HMME-SDT may be a potential therapeutic approach in the management of OS.

\section{Acknowledgements}

This study was supported in part by funding from Heilongjiang Provincial Health Bureau (grant no. 2012-690), Heilongjiang province science and technology plan and technological project (grant no. GC10C303-4), Harbin technological innovative personnel and special fund project (grant no. 2012RFXXS066).

\section{References}

1. Klein MJ and Siegal GP: Osteosarcoma: anatomic and histologic variants. Am J Clin Pathol 125: 555-581, 2006.

2. Tan ML, Choong PF and Dass CR: Osteosarcoma: conventional treatment vs. gene therapy. Cancer Biol Ther 8: 106-117, 2009.

3. Won KY,Lee CH, Kim YW and Park YK: Primary giant-cell-rich osteosarcoma of the urinary bladder: usefulness of osteocalcin and osteonectin immunohistochemical staining and literature review. Pathology 43: 161-164, 2011.

4. Shibaguchi H, Tsuru H and Kuroki M: Sonodynamic cancer therapy: a non-invasive and repeatable approach using low-intensity ultrasound with a sonosensitizer. Anticancer Res 31: 2425-2429, 2011.

5. Rosenthal I, Sostaric JZ and Riesz P: Sonodynamic therapy-a review of the synergistic effects of drugs and ultrasound. Ultrason Sonochem 11: 349-363, 2004.

6. Lv Y, Fang M, Zheng J, et al: Low-intensity ultrasound combined with 5-aminolevulinic acid administration in the treatment of human tongue squamous carcinoma. Cell Physiol Biochem 30: 321-333, 2012.

7. Barati AH, Mokhtari-Dizaji M, Mozdarani H, Bathaie SZ and Hassan ZM: Treatment of murine tumors using dual-frequency ultrasound in an experimental in vivo model. Ultrasound Med Biol 35: 756-763, 2009.

8. Li H, Fan H, Wang Z, Zheng $\mathrm{J}$ and Cao W: Potentiation of scutellarin on human tongue carcinoma xenograft by low-intensity ultrasound. PLoS One 8: e59473, 2013.

9. Bai WK, Shen E and Hu B: The induction of the apoptosis of cancer cell by sonodynamic therapy: a review. Chin J Cancer Res 24: 368-373, 2012.

10. Wang X, Liu Q, Wang Z, et al: Role of autophagy in sonodynamic therapy-induced cytotoxicity in S180 cells. Ultrasound Med Biol 36: 1933-1946, 2010. 
11. Jaruga E, Salvioli S, Dobrucki J, et al: Apoptosis-like, reversible changes in plasma membrane asymmetry and permeability and transient modifications in mitochondrial membrane potential induced by curcumin in rat thymocytes. FEBS Lett 433: 287-293, 1998.

12. Tang W, Liu Q, Zhang J, Cao B, Zhao P and Qin X: In vitro activation of mitochondria-caspase signaling pathway in sonodynamic therapy-induced apoptosis in sarcoma 180 cells. Ultrasonics 50: 567-576, 2010.

13. Duvshani-Eshet M, Benny O, Morgenstern A and Machluf M: Therapeutic ultrasound facilitates antiangiogenic gene delivery and inhibits prostate tumor growth. Mol Cancer Ther 6: 2371-2382, 2007.

14. Nie F, Xu HX, Lu MD, Wang Y and Tang Q: Anti-angiogenic gene therapy for hepatocellular carcinoma mediated by microbubble-enhanced ultrasound exposure: an in vivo experimental study. J Drug Target 16: 389-395, 2008.

15. Fuentes-Prior P and Salvesen GS: The protein structures that shape caspase activity, specificity, activation and inhibition. Biochem J 384: 201-232, 2004.

16. Ribe EM, Serrano-Saiz E, Akpan N and Troy CM: Mechanisms of neuronal death in disease: defining the models and the players. Biochem J 415: 165-182, 2008

17. Denecker G, Ovaere P, Vandenabeele P and Declercq W: Caspase-14 reveals its secrets. J Cell Biol 180: 451-458, 2008.

18. Martinon F and Tschopp J: Inflammatory caspases and inflammasomes: master switches of inflammation. Cell Death Differ 14: 10-22, 2007.

19. Lamkanfi M, Festjens N, Declercq W, Vanden Berghe T and Vandenabeele P: Caspases in cell survival, proliferation and differentiation. Cell Death Differ 14: 44-55, 2007.

20. Lipponen P: Apoptosis in breast cancer: relationship with other pathological parameters. Endocr Relat Cancer 6: 13-16, 1999.

21. Wang P, Xu CS, Xu J, Wang X and Leung AW: Hypocrellin B enhances ultrasound-induced cell death of nasopharyngeal carcinoma cells. Ultrasound Med Biol 36: 336-342, 2010.

22. Wang P, Xu C, Xia X, et al: Mitochondrial damage in nasopharyngeal carcinoma cells induced by ultrasound radiation in the presence of hypocrellin B. J Ultrasound Med 29: 43-50, 2010.

23. Tang W, Liu Q, Wang X, Wang P, Zhang J and Cao B: Potential mechanism in sonodynamic therapy and focused ultrasound induced apoptosis in sarcoma 180 cells in vitro. Ultrasonics 49: 786-793, 2009
24. He Y, Xia X, Xu C, et al: 5-Aminolaevulinic acid enhances ultrasound-induced mitochondrial damage in K562 cells. Ultrasonics 50: 777-781, 2010.

25. Wang XB, Liu QH, Mi N, et al: Sonodynamically induced apoptosis by protoporphyrin IX on hepatoma-22 cells in vitro. Ultrasound Med Biol 36: 667-676, 2010.

26. Dai S, Hu S and Wu C: Apoptotic effect of sonodynamic therapy mediated by hematoporphyrin monomethyl ether on C6 glioma cells in vitro. Acta Neurochir (Wien) 151: 1655-1661, 2009.

27. Yumita N, Umemura S, Magario N, Umemura $\mathrm{K}$ and Nishigaki R: Membrane lipid peroxidation as a mechanism of sonodynamically induced erythrocyte lysis. Int J Radiat Biol 69: 397-404, 1996.

28. Park JW, Ryter SW and Choi AM: Functional significance of apoptosis in chronic obstructive pulmonary disease. COPD 4: 347-353, 2007.

29. Schuler M and Green DR: Mechanisms of p53-dependent apoptosis. Biochem Soc Trans 29: 684-688, 2001.

30. Haupt S, Berger M, Goldberg Z and Haupt Y: Apoptosis-the p53 network. J Cell Sci 116: 4077-4085, 2003.

31. Martin DA and Elkon KB: Mechanisms of apoptosis. Rheum Dis Clin North Am 30: 441-454, 2004

32. Yumita N, Okudaira K, Momose $\mathrm{Y}$ and Umemura S: Sonodynamically induced apoptosis and active oxygen generation by gallium-porphyrin complex, ATX-70. Cancer Chemother Pharmacol 66: 1071-1078, 2010.

33. Juffermans LJ, Dijkmans PA, Musters RJ, Visser CA and Kamp O: Transient permeabilization of cell membranes by ultrasound-exposed microbubbles is related to formation of hydrogen peroxide. Am J Physiol Heart Circ Physiol 291: H1595-H1601, 2006.

34. Kumon RE, Aehle M, Sabens D, et al: Spatiotemporal effects of sonoporation measured by real-time calcium imaging. Ultrasound Med Biol 35: 494-506, 2009.

35. Hutcheson JD, Schlicher RK, Hicks HK and Prausnitz MR: Saving cells from ultrasound-induced apoptosis: quantification of cell death and uptake following sonication and effects of targeted calcium chelation. Ultrasound Med Biol 36: 1008-1021, 2010.

36. Tabuchi Y, Takasaki I, Zhao QL, et al: Genetic networks responsive to low-intensity pulsed ultrasound in human lymphoma U937 cells. Cancer Lett 270: 286-294, 2008. 